Proceedings of the $51^{\text {st }}$ Hawaii International Conference on System Sciences | 2018

\title{
Energy-Aware Scheduling of Conditional Task Graphs on NoC-Based MPSoCs
}

\author{
Umair Ullah Tariq*, Hui Wu* and Suhaimi Abd Ishak*† \\ ${ }^{*}$ The University of New South Wales, Australia \\ ${ }^{\dagger}$ Universiti Tun Hussein Onn, Malaysia \\ \{tariqu, huiw, sishak\}@cse.unsw.edu.au
}

\begin{abstract}
We investigate the problem of scheduling a set of tasks with individual deadlines and conditional precedence constraints on a heterogeneous Network on Chip (NoC)-based Multi-Processor System-on-Chip (MPSoC) such that the total expected energy consumption of all the tasks is minimized, and propose a novel approach. Our approach consists of a scheduling heuristic for constructing a single unified schedule for all the tasks and assigning a frequency to each task and each communication assuming continuous frequencies, an Integer Linear Programming (ILP)-based algorithm and a polynomial time heuristic for assigning discrete frequencies and voltages to tasks and communications. We have performed experiments on 16 synthetic and 4 real-world benchmarks. The experimental results show that compared to the state-of-theart approach, our approach using the ILP-based algorithm and our approach using the polynomial-time heuristic achieve average improvements of $31 \%$ and $20 \%$, respectively, in terms of energy reduction.
\end{abstract}

\section{Introduction}

Modern mobile systems such as robots and driverless cars require computationally powerful and energy-efficient hardware due to their complex functions and battery power constraint. MPSoC is an ideal architecture for those mobile systems due to its high performance and low power dissipation. Examples of commercial MPSoCs include Samsung Exynos 5422 SoC [1] and Zynq UltraScale+ MPSoC devices [3]. Samsung Exynos 5422 SoC powers the famous Samsung Galaxy smartphone series. Zynq UltraScale+ MPSoC devices have been used in robots. Typically an MPSoC consists of processors with different power and performance profiles. For example, Samsung Exynos $5422 \mathrm{SoC}$ consists of 4 highperformance ARM Cortex-A15 CPU, 4 low-power ARM Cortex-A7 CPU. Modern MPSoCs have a large number of processors and the number of processors on MPSoCs are expected to grow [10]. According to International Technology Roadmap for Semiconductors (ITRS), MPSoCs will integrate thousands of processors [15] by 2025. Therefore, the traditional bus-based on-chip communication is no longer feasible due to its poor scalability. NoC-based communication provides

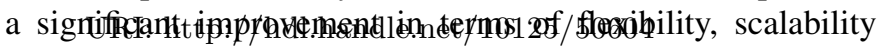

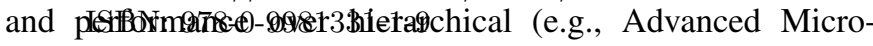
(CC BY-NC-ND 4.0) controller Bus Architecture and STBus) and traditional bus structures [23].

Mobile systems are battery powered. Although battery lifetimes have increased over the years, modern batteries are still far from meeting the needs of power-hungry mobile devices. Therefore, energy efficiency is a critical issue in mobile systems. One way to improve energy efficiency is to apply Dynamic Voltage and Frequency Scaling (DVFS). DVFS saves energy consumption by lowering the voltage/frequency of a processor/communication link when it is underutilized. For example, in order to reduce the energy consumption of a Nexus 4 Android smartphone on-demand governor scales the CPU frequency and voltage level based on CPU utilization every 50 millisecond [17]. In addition to processors, NoC communication links and routers also consume a large amount of on-chip energy. For Alpha 21364 processor [32], out of $125 \mathrm{~W}$ total on-chip power consumption, $23 \mathrm{~W}(20 \%)$ is consumed by NoC routers and links, and out of $23 \mathrm{~W}$, the NoC links consume $58 \%$ of the power. Therefore, it is important to take communication energy into account when mapping applications onto NoC-based MPSoCs.

In this paper, we target energy-efficient mobile embedded systems such as driver-less cars, robots and advanced combat helmets using a NoC-based MPSoC as the hardware platform. For those mobile systems, their complex functions such as object recognition and communication, are known at the design stage, and the embedded software is typically modelled as a set of tasks with conditional precedence constraints and individual deadlines. We investigate the problem of scheduling a set of tasks with conditional precedence constraints and individual deadlines on a heterogeneous NoC-based MPSoC such that the total expected processor and communication energy is minimized. The processors and NoC links are voltage scalable and can operate at a set of discrete voltage/frequency levels. We make the following major contributions:

1) We propose a novel offline task scheduling approach. Our approach consists of a task scheduling heuristic that constructs a single unified schedule for all the tasks and collectively assigns a frequency to each task and each communication assuming continuous frequencies, and an ILP-based algorithm and a polynomial-time heuristic for assigning a discrete frequency to each task and each communication. To the best of our knowledge, our approach is the first one that investigateagthe 7 problem $\mathrm{H} \stackrel{\text { II }}{\mathrm{CS}} \mathrm{S}^{\text {heduling a set of tasks and communications with }}$ 
conditional precedence constraints on NoC-based MPSoCs such that the total expected energy consumption is minimized.

2) We have performed experiments on 20 benchmarks. Compared to the state-of-the-art approach proposed by $\mathrm{Li}$ and $\mathrm{Wu}$ [24] that does not consider conditional precedence constraints, our approach using the ILP-based algorithm achieves an average improvement of $31 \%$ and a maximum improvement of $61 \%$, and our approach using the polynomial-time heuristic achieves an average improvement of $20 \%$ and a maximum improvement of $46 \%$. Furthermore, both our approach using the ILPbased algorithm and our approach using the polynomialtime heuristic run approximately three times faster than the state-of-the-art approach.

The rest of this paper is organized as follows. Section 2 gives an overview of the related work. Section 3 describes all the models, including the task model, the power models, and the MPSoC model. Section 4 presents our heuristic for task scheduling and frequency assignment assuming continuous frequencies for both processors and communications. Section 5 proposes an ILP-based algorithm and a polynomialtime heuristic for assigning discrete frequencies to tasks and communications. Section 6 presents our experimental results and analysis. Lastly, Section 7 concludes this paper.

\section{Related Work}

Several approaches have been proposed to minimize energy consumption for heterogeneous multi-processors systems. Gebotys et al. [12] investigate the problem of scheduling tasks onto heterogeneous processors such that total energy consumption is minimized. In their approach task mapping and scheduling are integrated with dynamic voltage scaling to maximize energy efficiency. Singh et al. [38] propose a contention-aware, energy efficient, duplication-based mixed integer programming (CEEDMIP) formulation for scheduling task graphs on NoC-based heterogeneous multiprocessors. The key idea of their approach is to duplicate some tasks to reduce the communication energy as well as traffic congestion. Zhang et al. [45] propose an ILP-based, energy-aware task mapping algorithm on heterogeneous multi-processors, and an evolutionary algorithm-based, energy-efficient task mapping heuristic. Cai et al. [6] propose an energy efficient approach for heterogeneous multi-processor mobile embedded systems. Their approach assigns discrete frequencies to tasks based on the critical path lengths of tasks. Lin et al. [25] propose an energy-efficient algorithm for heterogeneous MPSoC-based mobile devices. They integrate task mapping and scheduling with dynamic voltage scaling to reduce the energy consumption of mobile devices.

Huang et al. [21] propose a simulated annealing-based energy-aware task mapping algorithm on heterogeneous NoCbased MPSoCs. In their model, processors are assumed to be voltage scalable and NoC links operate at a fixed frequency. Mixed Integer Linear Programming (MILP) is used to assign voltages/frequencies to tasks. Shin et al. [37] consider a NoC model with voltage scalable links and propose a genetic algorithm for minimizing the communication energy of the
NoC by scaling the link voltages. Ghosh et al. [13] consider a model similar to that of Huang et al. [21] and propose an energy-aware task scheduling heuristic based on MILP relaxation and randomized rounding. Li et al. [24] assume a NoC model with voltage scalable links. They propose a task mapping algorithm and a genetic algorithm-based task voltage/frequency assignment algorithm. A detailed survey on approaches for multi-processor energy-efficient embedded computing is given in [31].

Only a few approaches have been proposed aiming at minimizing the energy consumption of tasks with conditional precedence constraints. Shin et al. [36] propose a scenariobased offline Non-Linear Programming (NLP) algorithm that assigns each task a speed for each scenario. The approach has an exponential time complexity as it constructs a separate schedule for each scenario. Wu et al. [41] propose an approach that employs the schedule table generated by [9] to identify the available slack time in the worst-case and propose a heuristic that assigns a frequency to each task. In [28] a heuristic is proposed to assign each task a speed based on the critical path length. The heuristic has an exponential time complexity in the worst case since it enumerates all the possible scenarios when computing the critical path lengths. Umair et al. [40] propose a task mapping algorithm for periodic CTGs, and propose an NLP-based algorithm and a heuristic to assign voltages/frequencies to tasks.

Energy efficiency is not only critical in mobile embedded systems but also important in cloud computing. In cloud data centers, efficient power management may reduce operation costs, increase system reliability and reduce adverse effects of large power consumption on environments. Many approaches have been proposed to minimize energy consumption in the data center. Hasan et al [19] formulate the problem of offline scheduling of jobs on the servers of a data center such that the total energy consumption is minimized, as a binary integer program. They also propose an online heuristic for the same problem. Sarood et al. [35] propose an Integer Linear Programming (ILP)-based scheduler to reduce energy consumption in data centers. Huai et al. [20] propose a load balancing algorithm combined with DVFS to significantly reduce the energy consumption of data center servers. Roukh et al. [34] argue that database management systems (DBMSs) are one of the major energy consumers in data centers, and propose a machine learning-based approach to reduce the energy consumption of nodes of database clusters when optimizing queries. $\mathrm{Xu}$ et al. [42] propose an energy-aware query optimization platform called PET for DBMSs. PET estimates the energy costs of queries offline and the evaluation engine of the DBMS configures PET parameters towards a desired energy/performance trade-off. Guo et al. [16] propose an energy efficient query processing framework in DBMSs. Their approach works out energy cost query plans and makes a trade-off between the performance and the energy plans. Authors in [18] and [14] discuss practices in detail to reduce the energy footprint of data centers. Mittal et al. [30] give a survey of power management techniques for data centers.

Our approach differs from all the previouథapprowahes in three major aspects. First, our approach considers conditional 


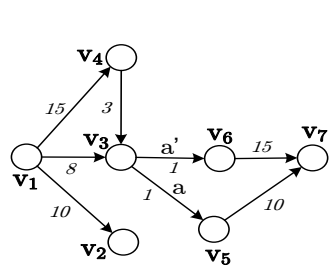

(a)

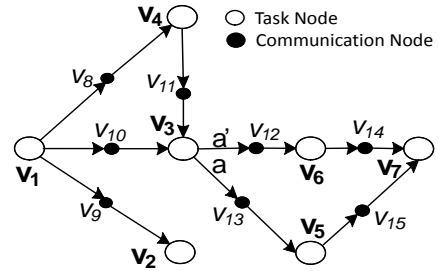

(b)
Fig. 1. (a) A CTG $G$ (b) An extended CTG $G_{e}$

precedence constraints. Second, our approach handles NoC and takes link contentions into account. Third, our approach collectively optimizes the frequencies of processors and NoC links aiming at minimizing the total expected energy consumption of the MPSoC.

\section{Models}

The target application is modelled by a conditional task graph (CTG) [39]. A CTG is a weighted directed acyclic graph $G(V, E, A, X)$ defined as follows. $V=\left\{v_{1}, v_{2}, \ldots, v_{n}\right\}$ is a set of tasks. Each task has an execution time represented by the number of clock cycles on each processor and a deadline $d_{i}$. All the tasks are non-preemptible. $E \subseteq V \times V$ is a set of directed edges each denoting the dependency between the two tasks. $A$ is a set of triplets $\left(e_{i}, c_{i}, p\left(c_{i}\right)\right)$, where $e_{i} \in E$, and $c_{i}$ and $p\left(c_{i}\right)$ represent the condition associated with $e_{i}$ and its probability [26], respectively. $X$ is a set of edge weights. An edge weight $\chi_{s} \in X$ of an edge $e_{s}=\left(v_{i}, v_{j}\right)$ represents the communication volume in bits from task $v_{i}$ to task $v_{j}$. Our task model is described in detail in [39]. The execution probability of each node $v_{i} \in V$ is represented by $p\left(v_{i}\right)$. We use algorithm presented in [26] to compute the probabilities.

The MPSoC has a set $P=\left\{p e_{1}, p e_{2}, \ldots, p e_{m}\right\}$ of $\mathrm{m}$ processors. We assume heterogeneous processors, where each processor $p e_{k} \in P$ is DVFS-enabled and can operate on a set $\left\{\left(V_{d d_{1}}, f_{1}\right), \ldots,\left(V_{d d_{n_{k}}}, f_{n_{k}}\right)\right\}$ of $n_{k}$ discrete voltagefrequency pairs. A matrix $N C$ represents the execution times in clock cycles of all the tasks in $G$ on different processors, where $N C(j, i)$ is the number of clock cycles of task $v_{i}$ on $p e_{j}$.

The dynamic power $P_{d_{k, i}}$ of a task $v_{i}$ on processor $p e_{k}$, dominated by discharging and charging of load capacitance due to gate switching, is given as $P_{d_{k, i}}=C_{e f f_{k, i}} V_{d_{d_{k, i}}}^{2} f_{k, i}$ [5], [4], where $C_{e f f_{k, i}}, V_{d d_{k, i}}$ and $f_{k, i}$ are the effective load switching capacitance, the supply voltage and the operating frequency, respectively. The execution time of a task $v_{i}$ on processor $p e_{k}$ operating at frequency $f_{k, i}$ is given as $t_{k, i}=$ $N C(k, i) / f_{k, i}$. The operating frequency $f$ is approximated by $f=\left(\left(1+K_{1}\right) V_{d d}+K_{2} V_{b s}-V_{t h_{1}}\right)^{\alpha} / K_{6} L_{d} V_{d d}$ [4], where $K_{1}, K_{2}, K_{6}$ and $V_{t h_{1}}$ are circuit dependent constants, $L_{d}$ is the logic depth, and $\alpha$ is velocity saturation imposed by the used technology $(1.4 \leq \alpha \leq 2)$. The total energy consumption $E_{k, i}$ of a task $v_{i}$ on $p e_{k}$ is computed as follows [4]:

$$
\begin{array}{r}
E_{k, i}=N C(k, i) C_{e f f_{k, i}} V_{d d_{k, i}}^{2}+L_{g}\left(V_{d d_{k, i}} K_{3} e^{K_{4} V_{d d_{k, i}}}\right. \\
\left.e^{K_{5} V_{b s}}+\left|V_{b s}\right| I_{j}\right) t_{k, i}
\end{array}
$$

We consider the 2D mesh NoC architecture, where each processor is associated with a router, and there are $N_{R}$ rows and $N_{C}$ columns. Every router has five ports with one port used to communicate with the associated processor, and the remaining four ports used to communicate with the neighboring routers. A link connecting two routers is called global link and a link connecting a router with its associated processor is referred to as a local link. All the links are full duplex. All the global links are identical and have same link width (also called bus width or the number of wires) $b_{w}$.

We only take into account the energy consumption of global links and neglect the energy consumption of the local links. In the rest of this paper, links refer to global links unless they are explicitly specified.

The NoC links can operate at a set $\left\{\left(V_{d d_{1}}, f_{1}\right), \ldots\right.$, $\left.\left(V_{d d_{F}}, f_{F}\right)\right\}$, of voltage-frequency pairs. In a $2 \mathrm{D}$ mesh, the Manhattan distance $\eta_{i, j}$ between two processors $p e_{i}$ and $p e_{j}$ is defined as follows: $\eta_{i, j}=\left|x_{i}-x_{j}\right|+\left|y_{i}-y_{j}\right|$, where $\left(x_{i}, y_{i}\right)$ and $\left(x_{j}, y_{j}\right)$ are the coordinates of $p e_{i}$ and $p e_{j}$, respectively.

The wormhole switching [27], [24] and deterministic $X Y$ routing are used. We do not scale router frequencies as adjusting router frequencies makes the problem too complex. We assume that router frequencies are fixed and commensurate with link frequencies as in [24].

Consider the message $e_{i}$ for a communication node. The time taken by $e_{i}$ on the links operating at frequency $f_{i}$ such that $e_{i}$ traverses the network without contention is calculated as follows [24]:

$$
t_{i}=\frac{\chi_{i}}{b_{w} f_{i}}
$$

We use the bit energy model given in [43], [29] for communication. Assume that the source node and the destination node of $e_{i}$ are mapped on processors $p e_{s}$ and $p e_{d}$, respectively. The energy of transmitting one bit of the message $e_{i}$ is $E_{b i t}=\left(\eta_{s, d}+1\right) E_{R b i t}+\eta_{s, d} E_{l b i t_{i}}$, where $E_{R b i t}$ is the energy consumption of one bit on one router, and $E_{l b i t_{i}}$ is the energy consumption of transmitting one bit on one link when all the links of $e_{i}$ operate at $f_{i}$. Thus, the energy consumption of transmitting $e_{i}$ on the links operating at frequency $f_{i}$ is calculated as follows:

$$
E_{\text {comm }_{i}}=\chi_{i}\left(\left(\eta_{s, d}+1\right) E_{R b i t}+\eta_{s, d} P_{i} /\left(f_{i} b_{w}\right)\right)
$$

where $P_{i}$ is the total power consumed in transmitting one bit when the links that $e_{i}$ traverses operate at frequency $f_{i} . P_{i}$ is the sum of the dynamic power $P_{d y n_{i}}$ and static power $P_{\text {stat }}$, $P_{i}=P_{d y n_{i}}+P_{\text {stat }}$ [4]. The static and dynamic powers depend on how links are implemented. The frequency $f_{i}$ is approximated by $f_{i}=\left(\left(1+K_{1}\right) V_{d d}+K_{2} V_{b s}-V_{t h_{1}}\right)^{\alpha} / K_{6} L_{d} V_{d d}$ [4].

\section{Task Mapping, Scheduling and Frequency As- signment}

In order to schedule tasks and communications in a unified way, we first transform a CTG $G$ into an extended CTG by adding an additional node for every edge in the original CTG. We refer to these additional nodes as communication nodes. The original nodes in $G$ are kept unchanged an $\phi_{\text {age }}$ tefforred to as task nodes. Specifically, for each edge $\left(v_{i}, v_{j}\right) \in G$, we add 
a communication node $v_{s}$, and replace $\left(v_{i}, v_{j}\right)$ by two directed edges $\left(v_{i}, v_{s}\right)$ and $\left(v_{s}, v_{j}\right)$. If $\left(v_{i}, v_{j}\right) \in G$ has a condition, $\left(v_{i}, v_{s}\right)$ has the same condition and probability. The extended graph is represented by $G_{e}\left(V+V^{*}, E^{\prime}, A^{\prime}\right)$, where $V$ is a set of task nodes, $V^{*}$ is a set of communication nodes, $E^{\prime}$ is a set of edges, and $A^{\prime}$ is a set of 3-tuples where each 3-tuple consists of an edge, the condition associated with the edge and probability of the condition. Figure 1(b) shows the extended graph $G_{e}$ of the CTG in Figure 1(a).

\subsection{Successor-Tree-Consistent Deadline}

Our offline scheduling algorithm schedules nodes using the priorities of task nodes and communication nodes. We extend the notion of successor-tree-consistent deadline [39] to NoC-based MPSoCs, and propose a priority scheme for nodes, where the priority of each node $v_{i}$ is its successortree-consistent deadline denoted by $d_{i}^{\prime}$. When computing the successor-tree-consistent deadline of each node, we assume that all the processors and NoC-links operate at the maximum frequencies. Furthermore, the original CTG is used rather than the extended graph $G_{e}$. Before defining the successor-treeconsistent deadline, we introduce the worst case set of a task. Let $I \operatorname{Pred}\left(v_{i}\right)$ and $I S u c c\left(v_{i}\right)$ be the sets of all the immediate predecessors and all the immediate successors of a task $v_{i}$, respectively.

Definition 1: The worst-case set of a task $v_{i}$, denoted by $W C S\left(v_{i}\right)$, is a set of tasks defined as follows:

1) If $v_{i}$ is a sink node, $W C S\left(v_{i}\right)=\emptyset$.

2) If $v_{i}$ is an OR-FORK node, $W C S\left(v_{i}\right)=\left\{v_{j}\right\} \cup$ $W C S\left(v_{j}\right)$, where $v_{j}$ is in $\operatorname{ISucc}\left(v_{i}\right)$ satisfying $d_{j}^{\prime}-\min _{\forall p e_{k} \in P}\left\{t_{k, j}\right\}=\min _{\forall v_{s} \in \operatorname{ISucc}\left(v_{i}\right)}\left\{d_{s}^{\prime}-\right.$ $\left.\min _{\forall p e_{k} \in P}\left\{t_{k, s}\right\}\right\}$.

3) If $v_{i}$ is an AND-FORK node, $W C S\left(v_{i}\right)=$ $\bigcup_{v_{s} \in I S u c c\left(v_{i}\right)}\left(W C S\left(v_{s}\right) \cup\left\{v_{s}\right\}\right)$.

Definition 2: Given a CTG G and a task $v_{i}$, the successor tree of a task $v_{i}$ is a weighted directed tree $\mathrm{ST}\left(\mathrm{G}, v_{i}\right)=\left(V^{\prime}\right.$, $\left.E^{\prime}, X^{\prime}\right)$ where $v^{\prime}=\left\{v_{i}\right\} \cup W C S\left(v_{i}\right), E^{\prime}=\left\{\left(v_{i}, v_{j}\right)\right.$ : $\left.v_{j} \in W C S\left(v_{i}\right)\right\}$, and $X^{\prime}=\left\{w_{i, j}^{\prime}\right.$ : if $v_{j}$ is an immediate successor of $v_{i}, w_{i, j}^{\prime}$ is equal to the edge weight of $\left(v_{i}, v_{j}\right)$ in $G$; otherwise, $\left.w_{i, j}^{\prime}=0\right\}$.

Definition 3: Given a task $v_{i}$, if $v_{i}$ is a sink task, its successor-tree-consistent deadline $d_{i}^{\prime}$ is equal to its preassigned deadline $d_{i}$. Otherwise, $d_{i}^{\prime}$ is the upper bound on the latest completion time of $v_{i}$ in any feasible schedule of the relaxed problem instance: a set $V^{\prime}=\left\{v_{i}\right\} \cup W C S\left(v_{i}\right)$ of tasks with the precedence constraints in the form of the successor tree $S T\left(G, v_{i}\right)$, where the deadline of each task $v_{j} \in W C S\left(v_{i}\right)$ is its successor-tree-consistent deadline, and the deadline of $v_{i}$ is its preassigned deadline, and the same MPSoC.

The successor-tree-consistent deadlines of all the tasks in $G$ are computed as follows. For each task $v_{i}$ in reverse topological order of $G$, if $v_{i}$ is a sink node, its successor-treeconsistent deadline $d_{i}^{\prime}$ is equal to its preassigned deadline $d_{i}$ and $W C S\left(v_{i}\right)$ is an empty set. Otherwise, compute $W C S\left(v_{i}\right)$, construct the successor tree of $v_{i}$, and do the following:

1) Partition the tasks in $W C S\left(v_{i}\right)$ into two disjoint sets $\mathrm{U}$ and $\mathbf{J}$. The set $\mathrm{U}$ consists of all the tasks in $W C S\left(v_{i}\right)$ each of which does not receive any data from $v_{i}$, and the set $\mathbf{J}$ contains all the tasks in $W C S\left(v_{i}\right)$ that are not in $\mathrm{U}$.

2) Sort all the tasks in $U$ in non-increasing order of their successor-tree-consistent deadlines.

3) Schedule each task $v_{j}$ in $\mathrm{U}$ on a processor that maximizes its start time.

4) Sort all the tasks in $J$ in non-increasing order of their successor-tree-consistent deadlines. For the tasks with the same successor-tree-consistent deadlines, sort them in non-increasing order of their edge weights.

5) Schedule each task $v_{j}$ in $\mathbf{J}$ on a processor that maximizes its start time.

6) Schedule $v_{i}$ on a processor that maximizes its completion time respecting the constraints specified by the successor tree of $v_{i}$.

7) Set $d_{i}^{\prime}$ to the completion time of $v_{i}$.

A communication node $v_{s}$ in the extended graph $G_{e}$ has a single child node $v_{j}$. Therefore, the successor-tree-consistent deadline of $v_{s}$ is $d_{s}^{\prime}=d_{j}^{\prime}-\min _{\forall p e_{k} \in P}\left\{t_{k, j}\right\}$, where the execution time $t_{k, j}$ of a task node $v_{j}$ is computed assuming the maximum processor frequency.

\subsection{Earliest Successor-Tree-Consistent Deadline First Algorithm}

In a CTG, the number of scenarios grows exponentially as the number of conditions increases. Therefore, it is not feasible to construct a separate schedule for each scenario. Our offline scheduling approach constructs a single unified schedule for all the scenarios by exploiting the mutual exclusion relations between communication and task nodes. Two nodes are said to be mutually exclusive in the graph $G_{e}$ if they cannot coexist in any scenario. For example, in Figure 1(a) $v_{5}$ and $v_{6}$ are mutually exclusive. Two mutually exclusive nodes can be allocated the same resource at the same time. In a CTG, two nodes are said to be concurrent if they are not reachable from each other in graph $G_{e}$ and are not mutually exclusive.

We propose an Earliest-Successor-Tree-consistent Deadline First (ESTDF) list scheduling algorithm assuming that all processors and links operate at the maximum frequencies. ESTDF is called by our main algorithm IOETCS described in the next subsection. It determines the order in which task nodes and communication nodes execute and captures this order by adding additional precedence constraints in the input graph $G$. The output of ESTDF is the input graph $G$ with the additional precedence constraints. Given a CTG $G$, a matrix $N C$ of worst-case clock cycles of tasks, a vector $X$ of communication volumes and a task-to-processor mapping Map, ESTDF works by constructing a set ReadySet containing the source nodes of $G$ and repeating the following steps until ReadySet is empty.

1) Select a node $v_{j}$ with the minimum successor-treeconsistent deadline from ReadySet.

2) Compute its ready time $r_{j}=\max \left\{\zeta_{l}: v_{l} \in\right.$ $\left.\operatorname{IPred}\left(v_{j}\right)\right\}$, where $\zeta_{l}$ is the finish time of the node $v_{l}$.

3) If $v_{j}$ is a communication node, compute its finish time $\zeta_{j}=r_{j}+t_{j}$, where $t_{j}$ is given in Equation (2), and insert unconditional directed edges in GPfrems 7 lyoto the communication nodes that are concurrent to $v_{j}$, have 
larger or equal successor tree consistent deadlines as compared to $v_{j}$ and traverse the same links that $v_{j}$ traverses.

4) If $v_{j}$ is a task node, compute its finish time $\zeta_{j}=r_{j}+$ $t_{k, j}$, and insert unconditional directed edges from $v_{j}$ to unscheduled nodes concurrent to $v_{j}$ and mapped on the same processor where $v_{j}$ is mapped.

5) Delete $v_{j}$ from ReadySet and insert all ready nodes in $G$ to ReadySet.

Consider the CTG in Figure 1(b) and the MPSoC in Figure 2(a) where all the processors are identical. The execution times of tasks at the maximum processor frequency are $t_{1,1}=$ $7, t_{1,2}=2, t_{1,3}=5, t_{1,4}=3, t_{1,5}=3, t_{1,6}=2, t_{1,7}=4$ time units. The communication times are $t_{8}=7, t_{9}=8, t_{10}=$ $6, t_{11}=5, t_{12}=4, t_{13}=5, t_{14}=7, t_{15}=9$ time units. All the tasks have a common deadline of 40 time units. Consider the task mapping in Figure 2(a). Based on this task mapping the input CTG $G_{e}$ shown in Figure 1(b) to ESTDF does not contain communication nodes $v_{11}, v_{14}$ and $v_{15}$. Furthermore edges $\left(v_{4}, v_{11}\right),\left(v_{11}, v_{3}\right),\left(v_{6}, v_{14}\right),\left(v_{14}, v_{7}\right)$, $\left(v_{5}, v_{15}\right),\left(v_{15}, v_{7}\right)$ in $G_{e}$ are replaced by $\left(v_{4}, v_{3}\right),\left(v_{6}, v_{7}\right)$ and $\left(v_{5}, v_{7}\right)$. Figure 2(b) gives an illustration of ESTDF scheduling algorithm for task mapping in Figure 2(a). Three communication nodes $v_{8}, v_{10}$ and $v_{9}$ become ready after $v_{1}$ is scheduled. Communication nodes $v_{8}$ and $v_{10}$ traverse the same link $l_{1}$. Since they are concurrent, they contend for $l_{1}$. ESTDF resolves this conflict by scheduling $v_{8}$ before $v_{10}$ as $v_{8}$ has a smaller successor-tree-consistent deadline than $v_{10}$. Since $v_{8}$ and $v_{10}$ are concurrent nodes, an edge is inserted from $v_{8}$ to $v_{10}$ to capture this order as shown in Figure 2(c). Notice that communication nodes $v_{12}$ and $v_{13}$ are allocated the same time slot even though both use the same link $l_{3}$. This is because both are mutually exclusive. No additional edges are inserted between $v_{12}$ and $v_{13}$ as they are not concurrent nodes.

\subsection{Iterative Offline Energy-Aware Task and Com- munication Scheduling Algorithm (IOETCS)}

We propose an iterative offline energy-aware task and communication scheduling algorithm (IOETCS), Algorithm 1, for a NoC-based MPSoC. IOETCS constructs a single unified schedule iteratively assuming continuous frequencies for both processors and links.

IOETCS repeats three major steps until all the nodes in $G_{e}$ are mapped and scheduled. First, it selects an unscheduled task node $v_{i} \in V$ with the smallest successor-tree-consistent deadline among all the unscheduled task nodes. Second, it initializes the initial energy consumption $E_{\text {ini }}$ of the schedule to infinity and repeats the following steps for every $p e_{k} \in P$ :

1) Tentatively assign $v_{i}$ to the processor $p e_{k}$ by $M a p[i] \leftarrow$ $k$ and construct a sub-graph $G_{s}\left(V_{s}+V_{s}^{*}, E_{s}\right)$ where $V_{s}$ is the set of all the mapped task nodes, $V_{s}^{*}$ is a set of communication nodes with both child and parent nodes mapped on different processors and $E_{s}$ is a set of all the edges where every edge in $E_{s}$ belongs to $E^{\prime}$ and both its head and tail nodes are in $V_{s}+V_{s}^{*}$. For each communication node $v_{s}$ whose parent node $v_{p}$ and child node $v_{c}$ are mapped on the same processor, insert a directed edge $\left(v_{p}, v_{c}\right)$ to $E_{s}$.

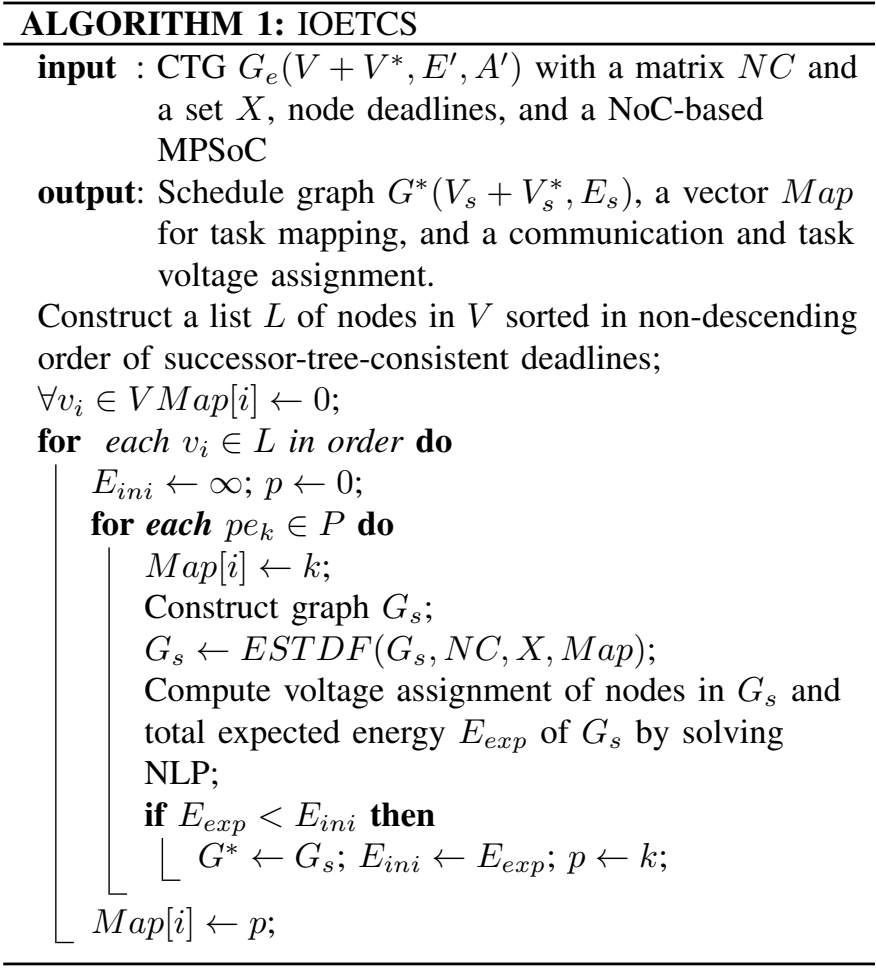

2) Call $G_{s} \leftarrow \operatorname{ESTDF}\left(G_{s}, N C, X, M a p\right)$ to construct a local schedule and capture the resource constraints introduced by the local schedule.

3) Given a task-to-processor mapping and a graph $G_{s}$, assign voltages/frequencies to task and communication nodes by solving a non-linear programming (NLP) problem. The objective of the NLP is to minimize the total expected energy consumption of graph $G_{s}$. The expected energy consumption is given as $E_{\text {exp }}=$ $\sum_{v_{i} \in V_{s}} p\left(v_{i}\right) E_{k, i}+\sum_{v_{i} \in V_{*}^{*}} p\left(v_{i}\right) E_{\text {comm }_{i}}$. The NLP problem is formulated as follows:

$$
\begin{aligned}
& \min \left\{E_{\text {exp }}\right\} \\
& \text { Subject To } \\
& \forall v_{i} \in V_{s} \quad t_{k, i}=\frac{N C(k, i) K_{6} L_{d} V_{d d_{k, i}}}{\left(\left(1+K_{1}\right) V_{d d_{k, i}}+K_{2} V_{b s}-V_{t h_{1}}\right)^{\alpha}} \\
& \forall v_{i} \in V_{s}^{*} \quad t_{i}=\frac{\chi_{i} K_{6} L_{d} V_{d d_{i}}}{b_{w}\left(\left(K V_{d d_{i}}+K_{2} V_{b s}-V_{t h_{1}}\right)^{\alpha}\right)} \\
& \forall v_{i} \in V_{s} \\
& \forall\left(v_{i}, v_{j}\right) \in E_{s} \wedge v_{i} \in V_{s} \quad \rho_{i}+t_{k, i} \leq \rho_{j} \\
& \forall\left(v_{i}, v_{j}\right) \in E_{s} \wedge v_{i} \in V_{s}^{*} \\
& \qquad \rho_{i} \geq 0 \\
& V_{d d_{k, \text { min }}} \leq t_{i} \leq \rho_{j}
\end{aligned}
$$

In Equation (5), $K=K_{1}+1$. The decision variables are, the start time $\rho_{i}$, the task node execution time $t_{k, i}$, the

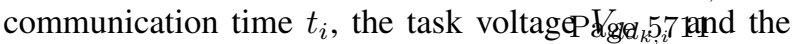
communication voltage $V_{d d_{i}} . V_{d d_{k, \text { max }}}$ and $V_{d d_{k, \text { min }}}$ are 


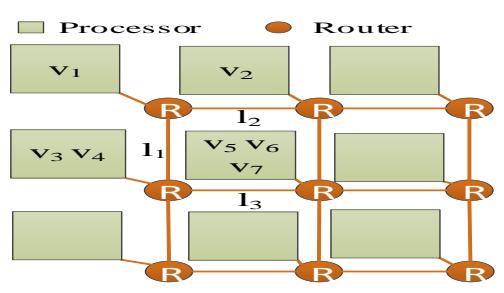

(a) Mapping

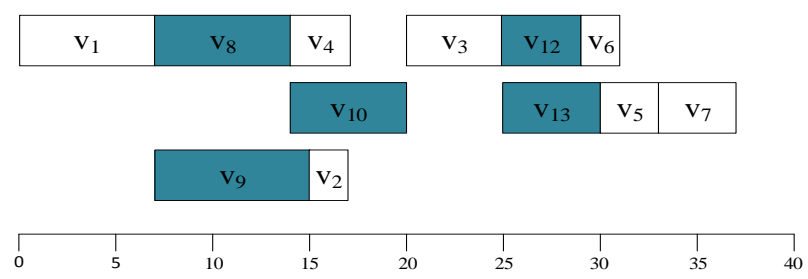

(b) Local Schedules

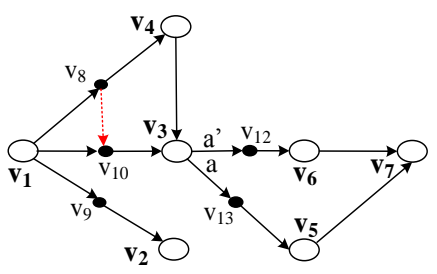

(c) Schedule Graph

Fig. 2. An illustrative example (a) Task-to-processor mapping (b) Local schedules constructed by ESTDF (b) Graph capturing the precedence and resource constraints

the minimum and the maximum supply voltages of the processor $p e_{k}$, respectively. Equations (4) and (5) are the task execution time and communication time constraints, respectively. Equation (6) is the deadline constraint, and the Equations (7), (8) are precedence constraints. Since the constraints and the objective function are convex, this NLP problem can be solved in polynomial time [44].

4) If the initial energy $E_{\text {ini }}$ is greater than $E_{\text {exp }}$, set $p \leftarrow k$, $G^{*} \leftarrow G_{s}$, and $E_{e x p} \leftarrow E_{i n i}$.

In the final step, IOETCS maps $v_{i}$ to processor $p$, and set $\operatorname{Map}[i] \leftarrow p$.

\section{Discrete Frequency Assignment}

Algorithm 1 constructs a graph $G^{*}\left(V_{s}+V_{s}^{*}, E_{s}\right)$ that captures the original precedence constraints and constraints introduced by the schedule, and assigns an optimal frequency/voltage to each node in $G^{*}$. However, the frequency/voltage level assigned to a node may not be a valid discrete frequency/voltage of the processor/link where the node is mapped. Therefore, we propose an ILP-based algorithm and a polynomial time heuristic for assigning a discrete frequency to each node.

\subsection{ILP-Based Algorithm}

The optimal frequency $f_{i}^{\text {opt }}$ of a communication node and the optimal frequency $f_{k, i}^{o p t}$ of a task node are computed as described in Section 4. We differentiate between the following two cases for each task or communication node $v_{i}$ :

1) If $v_{i}$ is a task node and its frequency $f_{k, i}^{o p t}$ is a discrete frequency of the processor $p e_{k}$ where $v_{i}$ is assigned, assign $f_{k, i}^{o p t}$ to $v_{i}$. If $v_{i}$ is a communication node and its frequency $f_{i}^{o p t}$ is equal to a discrete link frequency, assign $f_{i}^{o p t}$ to $v_{i}$.

2) If $v_{i}$ is a task node and its frequency $f_{k, i}^{o p t}$ is not a discrete frequency of the processor $p e_{k}$ where $v_{i}$ is assigned, find two frequencies $f_{k, i}^{o p t, u}$ and $f_{k, i}^{o p t, l}$ of the $p e_{k}$ where $v_{i}$ is assigned such that $f_{k, i}^{o p t}, u$ is the smallest discrete frequency of $p e_{k}$ larger than $f_{k, i}^{o p t}$ and $f_{k, i}^{o p t, l}$ is the largest discrete frequency of $p e_{k}$ smaller than $f_{k, i}^{o p t}$. Similarly, if $v_{i}$ is a communication node and its frequency $f_{i}^{o p t}$ is not a discrete link frequency, find two discrete frequencies $f_{i}^{o p t, l}$ and $f_{i}^{o p t, u}$ of communication links such that $f_{i}^{o p t, u}$ is the smallest discrete frequency of communication links larger than $f_{i}^{o p t}$ and $f_{i}^{o p t, l}$ is the largest discrete frequency of communication links smaller than $f_{i}^{o p t}$. Clearly, the optimal discrete frequency of $v_{i}$ must be either $f_{i}^{o p t, u}$ or $f_{i}^{o p t, l}$ for a communication node and either $f_{k, i}^{o p t, u}$ or $f_{k, i}^{o p t, l}$ for a task node.

We introduce a binary decision variable to select between $f_{i}^{o p t, u}$ and $f_{i}^{o p t, l}$ if $v_{i}$ is a communication node or between $f_{k, i}^{o p t, u}$ and $f_{k, i}^{o p t, l}$ if $v_{i}$ is a task node.

$$
x_{i}= \begin{cases}0 & \text { if } v_{i} \text { uses } f_{i}^{o p t, l} \text { or } f_{k, i}^{o p t, l} \\ 1 & \text { if } v_{i} \text { uses } f_{i}^{o p t, u} \text { or } f_{k, i}^{o p t, u}\end{cases}
$$

Let $V^{o p t}$ be a set of nodes that lie in Case 1. $V_{R}=V_{s} \backslash V^{o p t}$ is a set of task nodes and $V_{R}^{*}=V_{s}^{*} \backslash V^{o p t}$ is a set of communication nodes for which Case 2 holds. The expected energy consumption is now given as $E_{\text {exp }}=\sum_{v_{i} \in V_{R}}\left(\left(1-x_{i}\right) E_{k, i}^{o p t, l}+\right.$ $\left.x_{i} E_{k, i}^{o p t, u}\right) p\left(v_{i}\right)+\sum_{v_{i} \in V_{R}^{*}}\left(\left(1-x_{i}\right) E_{c o m m_{i}}^{o p t, l}+x_{i} E_{c o m m_{i}}^{o p t, u}\right) p\left(v_{i}\right)+$ $C$, where $E_{k, i}^{o p t, l}$ and $E_{k, i}^{o p t, u}$ (given in Equation (1)) are the energy consumptions of a task node $v_{i}$ on a processor $p e_{k}$ at the frequencies $f_{k, i}^{o p t, l}$ and $f_{k, i}^{o p t, u}$, respectively, $E_{c o m m_{i}}^{o p t, l}$ and $E_{\text {comm }}^{o p t, u}$ (given in Equation (3)) are the energy consumptions of a communication node $v_{i}$ when all the links on its routing path operate at the frequencies $f_{i}^{o p t, l}$ and $f_{i}^{o p t, u}$, respectively and $C$ is the sum of energy consumption of nodes in $V^{o p t}$. The ILP problem is formulated as follows:

$$
\begin{aligned}
& \min \left\{E_{\text {exp }}\right\} \\
& \text { Subject To } \\
& \forall v_{i} \in V_{R} \quad t_{k, i}=t_{k, i}^{\text {opt }, l}\left(1-x_{i}\right)+t_{k, i}^{\text {opt }, u} x_{i} \\
& \forall v_{i} \in V_{R}^{*} \quad t_{i}=t_{i}^{o p t, l}\left(1-x_{i}\right)+t_{i}^{o p t, u} x_{i} \\
& \forall v_{i} \in V_{R} \quad \rho_{i}+t_{k, i} \leq d_{i}^{\prime} \\
& \forall v_{i} \in V^{o p t}, V_{s} \quad \rho_{i}+t_{k, i}^{\text {opt }} \leq d_{i}^{\prime} \\
& \forall\left(v_{i}, v_{j}\right) \in E_{s} \wedge v_{i} \in V^{o p t}, V_{s} \quad \rho\left(v_{i}\right)+t_{k, i}^{o p t} \leq \rho_{j} \\
& \forall\left(v_{i}, v_{j}\right) \in E_{s} \wedge v_{i} \in V^{o p t}, V_{s}^{*} \quad \rho\left(v_{i}\right)+t_{i}^{o p t} \leq \rho_{j} \\
& \forall\left(v_{i}, v_{j}\right) \in E_{s} \wedge v_{i} \in V_{R} \quad \rho\left(v_{i}\right)+t_{k, i} \leq \rho_{j} \\
& \forall\left(v_{i}, v_{j}\right) \in E_{s} \wedge v_{i} \in V_{R}^{*} \quad \rho\left(v_{i}\right)+t_{i} \leq \rho_{j} \\
& \qquad \rho_{i} \geq 0 \quad
\end{aligned}
$$

The decision variables are task execution time $t_{k, i}$, communication time $t_{i}$, binary variable $x_{i}$ and start time $\rho_{i} . t_{k, i}^{o p t, l}$ and $t_{k, i}^{o p t, u}$ are the execution times of the task node $v_{i}$ on the processor $p e_{k}$ where $v_{i}$ is mapped at the frequencies $f_{k, l}^{o p t}$ and $f_{k, u}^{o p t}$, respectively. $t_{i}^{o p t, l}$ and $t_{i}^{o p t, u}$ are the eQgangunizcation times (given in Equation (2)) of the communication node 
$v_{i}$ when all the links of the communication path operate at the frequencies $f_{i}^{o p t, l}$ and $f_{i}^{o p t, u}$, respectively. $t_{k, i}^{o p t}$ is the execution time of the task node $v_{i}$ at frequency level $f_{k, i}^{o p t}$ and $t_{i}^{o p t}$ is the communication time of the communication node $v_{i}$ when all the links of the communication operate at the frequency $f_{i}^{o p t}$. Equation (12) defines the execution time of a task node. Equation (13) defines the communication time of a communication node. Equations (14) and (15) collectively define the deadline constraints, Equations (16), (17), (18) and (19) collectively define the precedence constraints.

\subsection{Heuristic Algorithm}

The ILP problem is a well-known NP-Complete problem. Therefore, the previous ILP-based algorithm is not scalable. Next, we propose a polynomial time heuristic to assign discrete frequencies to task and communication nodes. The heuristic uses the schedule constructed by IOETCS algorithm (Algorithm 1) and works as follows:

1) Compute the cuts of graph $G^{*}$ as follows:

- Create a copy $G^{\prime}$ of $G^{*}$ and repeat the following steps until $G^{\prime}$ is empty:

a) Create a cut containing all the source nodes with zero in-degree in $G^{\prime}$.

b) Remove all the source nodes and their incident edges from $G^{\prime}$.

2) For every node $v_{i} \in V_{s}+V_{s}^{*}$, if its optimal frequency computed by NLP is a discrete frequency, assign the optimal frequency to $v_{i}$. Otherwise, assign $f_{k, i}^{o p t, l}$ to $v_{i}$ if $v_{i}$ is a task node or $f_{i}^{o p t, l}$ to $v_{i}$ if $v_{i}$ is a communication node.

3) Construct a new local schedule using the new frequency such that the order between nodes remain the same as in the schedule used by the NLP-based algorithm.

4) If there is no late task node, the algorithm terminates. Otherwise, repeat the following steps until there is no late task node.

- Find the first late task node $v_{j}$ and repeat the following steps until $v_{j}$ is not late.

a) Find a set $B$ of nodes where every node $v_{z} \in$ $B$ satisfies the following two conditions. First, $v_{z}$ belongs to the set $\left\{v_{j}\right\} \cup \operatorname{Pred}\left(v_{j}\right)$, where $\operatorname{Pred}\left(v_{j}\right)$ is a set of predecessors of $v_{j}$. Second, the frequency of $v_{z}$ has not been adjusted before and $v_{z}$ has not been assigned an optimal discrete frequency by NLP.

b) For every node $v_{i} \in B$, compute its rank. The rank of $v_{i}$ is a 2-tuple $\left(g_{i}, \kappa_{i}\right)$ which reflects the impact of $v_{i}$ on shifting the late node $v_{j}$ to an earlier time. Let $C_{p}$ be a set of nodes of a cut containing $v_{i}, C_{p}^{\prime}$ be $C_{p} \cap B, F T_{j}^{o l d}$ the finish time of $v_{j}$ in the current schedule, $F T_{j}^{\text {new }}$ the finish time of $v_{j}$ after the frequencies of all the nodes in the set $C_{p}^{\prime}$ are increased by one level, and $F T_{j}^{n e w, i}$ the finish time of $v_{j}$ when the frequency of $v_{i}$ is increased by one level. The normalized time gain $g_{i}$ of the cut
TABLE I

CHARACTERISTICS OF BENCHMARKS WITHOUT CONDITIONAL PRECEDENCE CONSTRAINTS

\begin{tabular}{|c|c|c|c|c|c|}
\hline BM & $a / b / D$ & Dim & BM & $a / b / D$ & Dim \\
\hline TG 1 & $17 / 19 / 1.4$ & $4 \times 5$ & TG 2 & $20 / 24 / 0.77$ & $5 \times 4$ \\
TG 2 & $15 / 11 / 0.98$ & $4 \times 5$ & TG 4 & $16 / 12 / 0.89$ & $5 \times 4$ \\
TG 5 & $27 / 28 / 2.4$ & $6 \times 5$ & TG 6 & $27 / 35 / 2.7$ & $6 \times 5$ \\
TG 7 & $27 / 39 / 2.9$ & $6 \times 5$ & TG 8 & $30 / 40 / 3.45$ & $6 \times 6$ \\
\hline
\end{tabular}

TABLE II

Characteristics of Benchmarks with Conditional Precedence CONSTRAINTS.

\begin{tabular}{|c|c|c|c|c|c|}
\hline BM & $x / y / z / D$ & Dim & BM & $x / y / z / D$ & Dim \\
\hline CTG1 & $17 / 2 / 6 / 0.74$ & $3 \times 3$ & CTG2 & $20 / 1 / 2 / 1.06$ & $3 \times 3$ \\
CTG3 & $15 / 2 / 4 / 0.723$ & $3 \times 3$ & CTG4 & $17 / 2 / 6 / 0.93$ & $3 \times 3$ \\
CTG5 & $30 / 4 / 11 / 1.73$ & $3 \times 3$ & CTG6 & $35 / 3 / 8 / 3.101$ & $3 \times 2$ \\
CTG7 & $33 / 5 / 15 / 3.7128$ & $3 \times 2$ & CTG8 & $31 / 3 / 9 / 3.69$ & $3 \times 2$ \\
\hline
\end{tabular}

containing $v_{i}$, is given as $g_{i}=\frac{F T_{j}^{o l d}-F T_{j}^{\text {new }}}{E_{T}+E_{C}}$, where $E_{T}=\sum_{v_{i} \in C_{p}^{\prime}, V_{s}}\left(E_{k, i}^{o p t, u}-E_{k, i}^{o p t, l}\right) p\left(v_{i}\right)$ and $E_{C}=\sum_{v_{i} \in C_{p}^{\prime}, V_{s}^{*}}\left(E_{i}^{o p t, u}-E_{i}^{o p t, l}\right) p\left(v_{i}\right)$. The normalized time gain $\kappa_{i}$ of $v_{i}$ is computed as:

$$
\kappa_{i}= \begin{cases}\frac{F T_{j}^{o l d}-F T_{j}^{n e w, i}}{\left(E_{i}^{o p t, u}-E_{i}^{o p t, l}\right) p\left(v_{i}\right)} & v_{i} \text { is commun- } \\ \frac{F T_{j}^{o l d}-F T_{j}^{n e w, i}}{\left(E_{k, i}^{o p t, u}-E_{k, i}^{o p t, l}\right) p\left(v_{i}\right)} & \text { ication node }\end{cases}
$$

c) Select a node with the highest rank by comparing ranks lexicographically. Adjust its frequency to $f_{i}^{o p t, u}$ if $v_{i}$ is a communication node or $f_{k, i}^{o p t, u}$ if $v_{i}$ is a task node. Update the schedule.

\section{PERFORMANCE EVALUATION}

In this section, we use IOETCS-ILP and IOETCS-Heuristic to denote our approach using the ILP-based algorithm and the heuristic, respectively, for assigning a discrete frequency to each task and each communication. To demonstrate the effectiveness of IOETCS-ILP and IOETCS-Heuristic, we compare them with three approaches. The first approach is $\mathrm{Li}-\mathrm{Wu}$ approach, a state-of-art approach for unconditional task graph model proposed in [24]. The second approach ILP-vpv-flv that is the same as IOETCS-ILP except that the NLP and ILP algorithms are modified such that they only scale processors frequencies/voltages and assign the maximum link frequency to all communication nodes. The third approach is ILP-fpvvlv that is the same as IOETCS-ILP except the NLP and ILP algorithms are modified such that they only scale the voltages of links and assign the maximum processor frequencies to task nodes.

\subsection{Simulation Setup}

We use the same experimental setup as in [11], [7], [4]. The technology parameters are taken from [7]. We use two types of processors in our experiments, Type 1 and Type 2, modelled after the processors in [7] and [8], respectively. The configuration for NoC links are adopted from [24]. The execution times in cycles of tasks are ramgdenglyzgenerated within $[10,100] \times 10^{6}$ and $[5,10] \times 10^{6}$, respectively. 


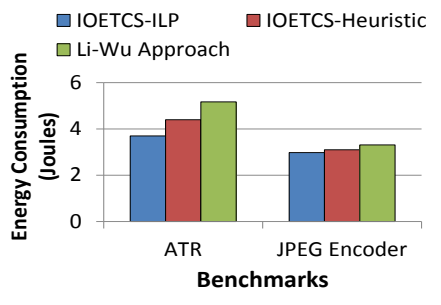

(a) Energy Consumption

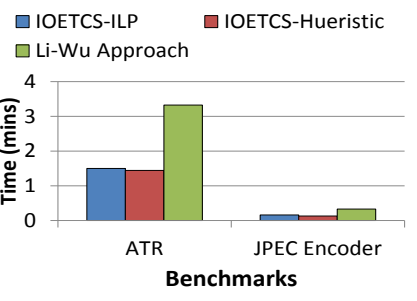

(b) Running Time

Fig. 3. Comparison of real-world benchmarks without conditional precedence constraints

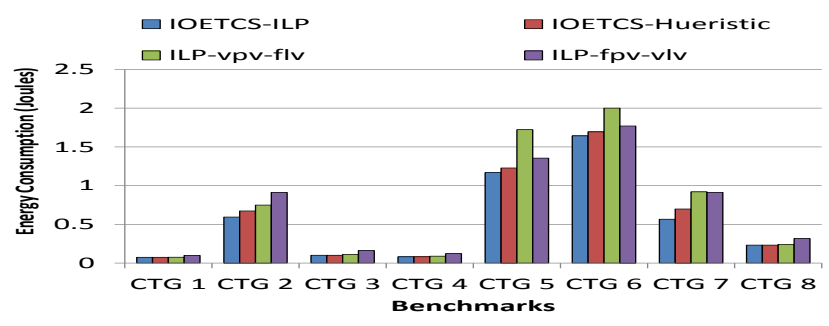

(a) Energy consumption

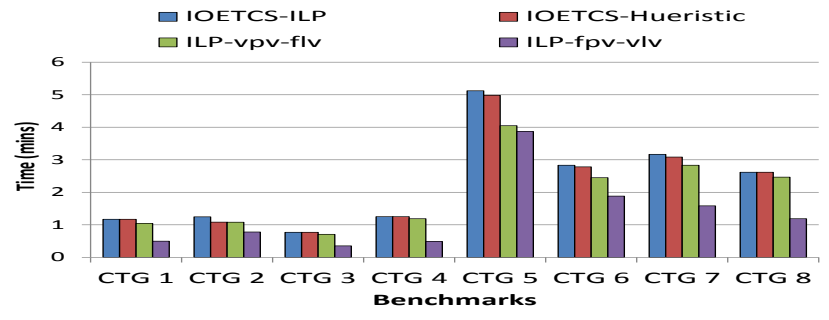

(b) Running time

Fig. 4. Comparison of eight benchmarks in Table II

The communication volumes are generated randomly within $[80,800] \times 10^{6}$ in bits. The deadline for each application is set to twice the makespan of the schedule of the application constructed by IOETCS algorithm assuming the maximum processors frequencies, the maximum links frequencies and a common deadline of 300 seconds for all the tasks so that there is reasonable slack for energy reduction. All the approaches are implemented in Matlab version R2015a. We use fmincon, quadprog and intlinprog solvers to solve the NLP, quadratic programming and ILP problems, respectively. The hardware platform consists of Intel(R) Core(TM) i5-4570 CPU with a clock frequency of $3.20 \mathrm{GHz}, 8.00 \mathrm{~GB}$ memory, and $3 \mathrm{MB}$ caches.

\subsection{Results and Discussion}

6.2.1 Experiments with conditional task graphs: In the first set of experiments we choose eight benchmarks and their details are given in Table II where $x / y / z / D$ stands for the number of tasks, the number of OR-FORK tasks, the number of conditions and the deadline of the application in seconds, respectively. The column with heading Dim represents NoC dimensions. The benchmarks in Table II are the same benchmarks used in [26].

IOETCS-ILP achieves an average improvement of $31 \%$, a maximum improvement of $62 \%$ for CTG 7 and a minimum

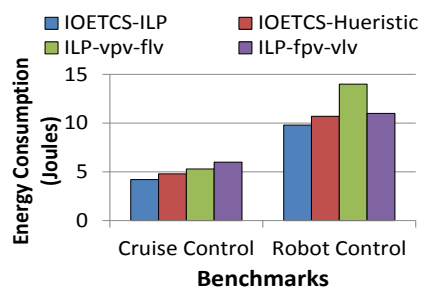

(a) Energy consumption

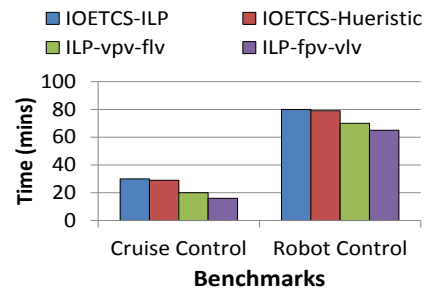

(b) Running time
Fig. 5. Comparison of real-world benchmarks with conditional precedence constraints

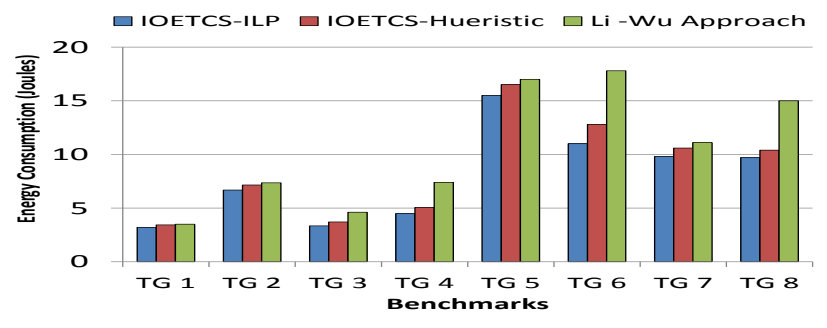

(a) Energy consumption

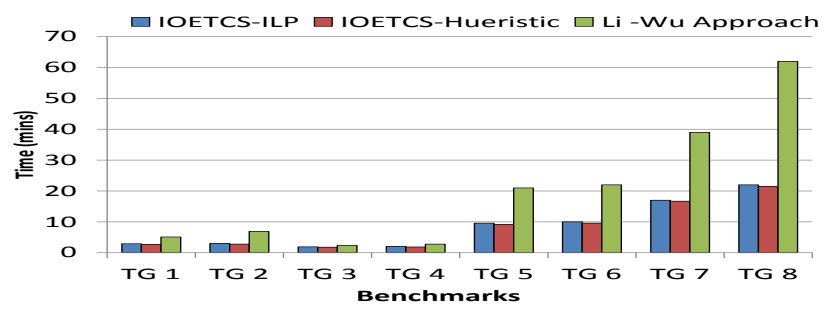

(b) Running time

Fig. 6. Comparison of eight benchmarks in Table I

improvement of $1.03 \%$ for CTG 1 over ILP-vpv-flv. It achieves an average improvement of $27 \%$, a maximum improvement of $61 \%$ for CTG 3 and a minimum improvement of $7.9 \%$ for CTG 6 over ILP-fpv-vlv. IOETCS-Heuristic achieves an average improvement of $23 \%$, a maximum improvement of $40 \%$ for CTG 5 and a minimum improvement of $1.3 \%$ for CTG 1 in comparison to ILP-vpv-flv. It achieves an average improvement of $18 \%$, a maximum improvement of $61 \%$ for CTG 3 and a minimum improvement of $4 \%$ for CTG 6 over ILP-fpvvlv. We observe that ILP-vpv-flv performs significantly better in terms of energy consumption if the computation energy dominates the total energy, and ILP-fpv-vlv performs better if communication energy dominates the total energy. CTG 5, 6 and 7 favour ILP-fpv-vlv as the communication volumes for these benchmarks are significantly larger than the execution times of task nodes. Both IOETCS-ILP and IOETCS-Heuristic distribute slacks efficiently between communication nodes and task nodes and thus perform significantly better than ILP-vpvflv and ILP-fpv-vlv. In terms of running time both ILP-vpvflv and ILP-fpv-vlv run slightly faster than IOETCS-ILP and IOETCS-Heuristic. This is because the search space of ILPvpv-flv and ILP-fpv-vlv is smaller as compared to IOETCSILP and IOETCS-Heuristic. ILP-vpv-flv only scales processor voltages and ILP-fpv-vlv only scales link volpages.5Whereas, IOETCS-ILP and IOETCS-Heuristic scale both the processor 
voltages and the link voltages.

We choose two real-world benchmarks vehicle cruise controller [33] and Robot control [2] that are the task graphs of actual applications. These benchmarks are executed on $3 \times 3$ NoC where the processors are selected randomly as either Type 1 or Type 2. Both IOETCS-ILP and IOETCS-Heuristic perform significantly better than ILP-vpv-flv and ILP-fpvvlv in terms of energy consumption. In terms of running time IOETCS-ILP and IOETCS-Heuristic take longer time compared to ILP-vpv-flv and ILP-fpv-vlv. The reason is that IOETCS algorithm cannot find a feasible solution for some sub-problems, and thus the solver takes a longer time to converge.

\subsubsection{Experiments with non-conditional task graphs:}

To demonstrate the effectiveness of our approach on task graphs without conditional precedence constraints, we have conducted a second set of experiments. We choose eight task graphs (TG) and their details are given in Table I where $\mathrm{a} / \mathrm{b} / \mathrm{D}$ stand for the number of tasks, the number of edges and the deadline of the application in seconds, respectively. The column with the heading Dim represents NoC dimensions. The benchmarks in Table II are the same benchmarks used in [26] except that all the edges are treated as unconditional edges. Figure 6(a) gives a comparison of 8 benchmarks in Table I in terms of energy consumption where all the processors are of Type 1. IOETCS-ILP achieves an average improvement of $31 \%$, a maximum improvement of $61 \%$ for TG 6 and a minimum improvement of $9 \%$ for TG 1 over $\mathrm{Li}-\mathrm{Wu}$ approach. IOETCS-Heuristic achieves an average improvement of $20 \%$, a maximum improvement of $46 \%$ for TG 4 and a minimum improvement of $2 \%$ for TG 1 over Li-Wu approach. We observe that $\mathrm{Li}-\mathrm{Wu}$ approach makes very poor mapping decisions for heterogeneous processors. The benchmarks TG 3, TG 4, TG 6 and TG 8 are executed on MPSoCs where the processors are randomly selected as either Type 1 or Type 2 . The reason for poor performance of $\mathrm{Li}-\mathrm{Wu}$ approach is that it does not take into account the energy profiles of processors when making mapping decisions. The benchmarks TG 1, TG 2, TG 5 and TG 8 are executed on MPSoCs with homogeneous processors (Type 1). As a result, Li-Wu approach performs considerably better. In terms of running time, IOETCS-ILP and IOETCSHeuristic run approximately three times faster than $\mathrm{Li}-\mathrm{Wu}$ approach. The major reason is that the genetic algorithm takes significantly longer time as it constructs a new schedule for each candidate solution using ETFGBF.

We have chosen two real-world benchmarks JPEG encoder [22] and Automatic Target Recognition (ATR) [24]. JPEG encoder is executed on a $3 \times 3$ MPSoC and ATR is executed on a $4 \times 5$ MPSoC. The processors are randomly selected as either Type 1 or Type 2. For both benchmarks, IOETCS-ILP and IOETCS-Heuristic outperform Li-Wu approach in terms of both running time and energy consumption.

We observe that the energy consumption of the schedules produced by IOETCS-Heuristic are close to those of the schedules produced by IOETCS-ILP. IOETCS-ILP achieves the average improvement of $11 \%$ over IOETCS-Heuristic in terms of energy consumption for all the problem instances. In terms of running time, IOETCS-Heuristic runs slightly faster than IOETCS-ILP.

\section{Conclusion}

We investigate the problem of energy-aware mapping and scheduling of tasks and communications with conditional precedence constraints and individual deadlines on a heterogeneous NoC-based MPSoC and propose a novel approach. Our approach reduces the total expected energy consumption by collectively optimizing the voltages/frequencies of processors and NoC links. The IOETCS algorithm maps tasks to processors and serializes communications that use same communication links. It constructs a unified schedule and assigns voltages/frequencies to tasks and communications collectively assuming continuous voltages/frequencies. The IOETCS algorithm significantly narrows down the search space for our ILP-based algorithm and our heuristic for assigning discrete frequencies/voltages to tasks and communications. The experimental results show that in terms of energy consumption, our approach using either ILP or heuristic outperforms the state-of-the-art approach proposed by $\mathrm{Li}$ and $\mathrm{Wu}$ [24] that considers only unconditional task graphs. Compared to the state-of-the-art approach, our ILP-based approach achieves an average improvement of $31 \%$, a maximum improvement of $61 \%$ and a minimum improvement of $9 \%$, and our heuristicbased approach achieves an average improvement of $20 \%$, a maximum improvement of $46 \%$ and a minimum improvement of $2 \%$. In terms of running time, our approach is approximately 3 times faster than the state-of-the-art approach.

\section{References}

[1] "Mobile processor exynos 5 octa (5422)," http://www.samsung.com/ semiconductor/minisite/Exynos/Solution/MobileProcessor/Exynos_5_ Octa_5422.html, accessed: 2017-09-4.

[2] "Standard task graph," URLhttp://www.kasahara.elec.waseda.ac.jp, accessed: 2017-09-4.

[3] "Zynq ultrascale+ mpsocs," https://www.xilinx.com/products/ silicon-devices/soc/zynq-ultrascale-mpsoc.html, accessed: 2017-09-4.

[4] A. Andrei, P. Eles, Z. Peng, M. T. Schmitz, and B. M. Al Hashimi, "Energy optimization of multiprocessor systems on chip by voltage selection," IEEE Transactions on Very Large Scale Integration (VLSI) Systems, vol. 15, no. 3, pp. 262-275, 2007.

[5] T. D. Burd and R. W. Brodersen, "Energy efficient cmos microprocessor design," in Proceedings of the Twenty-Eighth Hawaii International Conference on System Sciences, vol. 1. IEEE, 1995, pp. 288-297.

[6] Y. Cai, M. T. Schmitz, B. M. Al-Hashimi, and S. M. Reddy, "Workloadahead-driven online energy minimization techniques for battery-powered embedded systems with time-constraints," ACM Transactions on Design Automation of Electronic Systems (TODAES), vol. 12, no. 1, p. 5, 2007.

[7] G. Chen, K. Huang, and A. Knoll, "Energy optimization for real-time multiprocessor system-on-chip with optimal dvfs and dpm combination," ACM Transactions on Embedded Computing Systems (TECS), vol. 13, no. $3 \mathrm{~s}$, p. $111,2014$.

[8] K. Choi, R. Soma, and M. Pedram, "Fine-grained dynamic voltage and frequency scaling for precise energy and performance tradeoff based on the ratio of off-chip access to on-chip computation times," IEEE transactions on computer-aided design of integrated circuits and systems, vol. 24, no. 1, pp. 18-28, 2005.

[9] P. Eles, K. Kuchcinski, Z. Peng, A. Doboli, and P. Pop, "Scheduling of conditional process graphs for the synthesis of embedded systems," in

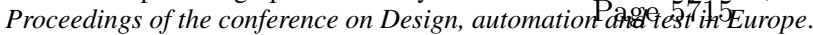
IEEE Computer Society, 1998, pp. 132-139. 
[10] M. Engel and O. Spinczyk, "A radical approach to network-on-chip operating systems," in System Sciences, 2009. HICSS'09. 42nd Hawaii International Conference on. IEEE, 2009, pp. 1-10.

[11] Y. Ge, Y. Zhang, P. Malani, Q. Wu, and Q. Qiu, "Low power task scheduling and mapping for applications with conditional branches on heterogeneous multi-processor system," Journal of Low Power Electronics, vol. 8, no. 5, pp. 535-551, 2012.

[12] C. H. Gebotys and R. J. Gebotys, "Power minimization in heterogeneous processing," in Proceedings of the Twenty-Ninth Hawaii International Conference on System Sciences, 1996., vol. 1. IEEE, 1996, pp. 330337.

[13] P. Ghosh, A. Sen, and A. Hall, "Energy efficient application mapping to noc processing elements operating at multiple voltage levels," in Proceedings of the 2009 3rd ACM/IEEE International Symposium on Networks-on-Chip. IEEE Computer Society, 2009, pp. 80-85.

[14] Q. Gu, P. Lago, H. Muccini, and S. Potenza, "A categorization of green practices used by dutch data centers," Procedia Computer Science, vol. 19, pp. 770-776, 2013

[15] G. Guindani and F. G. Moraes, "Achieving qos in noc-based mpsocs through dynamic frequency scaling," in 2013 International Symposium on System on Chip (SoC). IEEE, 2013, pp. 1-6.

[16] B. Guo, J. Yu, B. Liao, D. Yang, and L. Lu, "A green framework for dbms based on energy-aware query optimization and energy-efficient query processing," Journal of Network and Computer Applications, vol. 84, pp. 118-130, 2017.

[17] J. Guo and M. Potkonjak, "Coarse-grained learning-based dynamic voltage frequency scaling for video decoding," in 26th International Workshop on Power and Timing Modeling, Optimization and Simulation (PATMOS), 2016. IEEE, 2016, pp. 84-91.

[18] R. Harmon, H. Demirkan, N. Auseklis, and M. Reinoso, "From green computing to sustainable it: Developing a sustainable service orientation," in 43rd Hawaii International Conference on System Sciences (HICSS), 2010. IEEE, 2010, pp. 1-10.

[19] C. Hasan and Z. J. Haas, "Deadline-aware energy management in data centers," in IEEE International Conference on Cloud Computing Technology and Science (CloudCom), 2016. IEEE, 2016, pp. 79-84.

[20] W. Huai, Z. Qian, X. Li, G. Luo, and S. Lu, "Energy aware task scheduling in data centers." JoWUA, vol. 4, no. 2, pp. 18-38, 2013.

[21] J. Huang, C. Buckl, A. Raabe, and A. Knoll, "Energy-aware task allocation for network-on-chip based heterogeneous multiprocessor systems," in 19th Euromicro International Conference on Parallel, Distributed and Network-Based Processing (PDP), 2011. IEEE, 2011, pp. 447-454.

[22] J. In, S. Shirani, and F. Kossentini, "Jpeg compliant efficient progressive image coding," in Proceedings of the IEEE International Conference on Acoustics, Speech and Signal Processing, 1998., vol. 5. IEEE, 1998, pp. 2633-2636.

[23] H. G. Lee, N. Chang, U. Y. Ogras, and R. Marculescu, "On-chip communication architecture exploration: A quantitative evaluation of pointto-point, bus, and network-on-chip approaches," ACM Transactions on Design Automation of Electronic Systems (TODAES), vol. 12, no. 3, p. $23,2007$.

[24] D. Li and J. Wu, "Energy-efficient contention-aware application mapping and scheduling on noc-based mpsocs," Journal of Parallel and Distributed Computing, vol. 96, pp. 1-11, 2016.

[25] X. Lin, Y. Wang, Q. Xie, and M. Pedram, "Task scheduling with dynamic voltage and frequency scaling for energy minimization in the mobile cloud computing environment," IEEE Transactions on Services Computing, vol. 8, no. 2, pp. 175-186, 2015.

[26] M. Lombardi, M. Milano, M. Ruggiero, and L. Benini, "Stochastic allocation and scheduling for conditional task graphs in multi-processor systems-on-chip," Journal of scheduling, vol. 13, no. 4, pp. 315-345, 2010.

[27] Z. Lu, "Using wormhole switching for networks on chip: Feasibility analysis and microarchitecture adaptation," Ph.D. dissertation, KTH, 2005.

[28] P. Malani, P. Mukre, Q. Qiu, and Q. Wu, "Adaptive scheduling and voltage scaling for multiprocessor real-time applications with nondeterministic workload," in Proceedings of the conference on Design, automation and test in Europe. ACM, 2008, pp. 652-657.

[29] C. Marcon, N. Calazans, F. Moraes, A. Susin, I. Reis, and F. Hessel, "Exploring noc mapping strategies: an energy and timing aware technique," in Proceedings of the conference on Design, Automation and Test in Europe-Volume 1. IEEE Computer Society, 2005, pp. 502-507.

[30] S. Mittal, "Power management techniques for data centers: A survey," arXiv preprint arXiv:1404.6681, 2014.

[31] _ "A survey of techniques for improving energy efficiency in embedded computing systems," International Journal of Computer Aided Engineering and Technology, vol. 6, no. 4, pp. 440-459, 2014.

[32] S. S. Mukherjee, P. Bannon, S. Lang, A. Spink, and D. Webb, "The alpha 21364 network architecture," in Hot Interconnects 9, 2001. IEEE, 2001, pp. 113-117.

[33] P. Pop, Scheduling and communication synthesis for distributed real-time systems. Department of Computer and Information Science, Linköpings universitet, 2000

[34] A. Roukh, L. Bellatreche, N. Tziritas, and C. Ordonez, "Energy-aware query processing on a parallel database cluster node," in Algorithms and Architectures for Parallel Processing. Springer, 2016, pp. 260-269.

[35] O. Sarood, A. Langer, A. Gupta, and L. Kale, "Maximizing throughput of overprovisioned hpc data centers under a strict power budget," in Proceedings of the International Conference for High Performance Computing, Networking, Storage and Analysis. IEEE Press, 2014, pp. 807-818.

[36] D. Shin and J. Kim, "Power-aware scheduling of conditional task graphs in real-time multiprocessor systems," in Proceedings of the 2003 international symposium on Low power electronics and design. ACM, 2003, pp. 408-413.

[37] — "Communication power optimization for network-on-chip architectures," Journal of Low Power Electronics, vol. 2, no. 2, pp. 165-176, 2006.

[38] J. Singh, S. Betha, B. Mangipudi, and N. Auluck, "Contention aware energy efficient scheduling on heterogeneous multiprocessors," IEEE Transactions on Parallel and Distributed Systems, vol. 26, no. 5, pp. $1251-1264,2015$

[39] U. U. Tariq and H. Wu, "Energy-aware scheduling of conditional task graphs with deadlines on mpsocs," in IEEE 34th International Conference on Computer Design (ICCD), 2016. IEEE, 2016, pp. 265272

[40] — "Energy-aware scheduling of periodic conditional task graphs on mpsocs," in Proceedings of the 18th International Conference on Distributed Computing and Networking. ACM, 2017, p. 13.

[41] D. Wu, B. M. Al-Hashimi, and P. Eles, "Scheduling and mapping of conditional task graph for the synthesis of low power embedded systems," IEE Proceedings-Computers and Digital Techniques, vol. 150, no. 5, pp. 262-273, 2003 .

[42] Z. Xu, Y.-C. Tu, and X. Wang, "Pet: reducing database energy cost via query optimization," Proceedings of the VLDB Endowment, vol. 5, no. 12, pp. 1954-1957, 2012.

[43] T. T. Ye, G. D. Micheli, and L. Benini, "Analysis of power consumption on switch fabrics in network routers," in Proceedings of the 39th annual Design Automation Conference. ACM, 2002, pp. 524-529.

[44] A. N. Yurii Nesterov, Interior Point Polynomial Algorithms in Convex Programming. SIAM, 1987

[45] W. Zhang, E. Bai, H. He, and A. M. Cheng, "Solving energy-aware real-time tasks scheduling problem with shuffled frog leaping algorithm

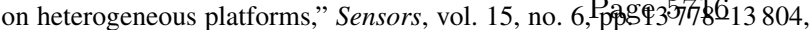
2015. 